\title{
A Systematic Scoping Review Protocol of the Impact of Mutual Care on Individuals with Learning Disabilities and their Ageing Carers in the United Kingdom
}

\author{
Mahon A, Vseteckova J*, Tilley E, Pappas Y and Randhawa G \\ Faculty of Wellbeing, Education and Language Studies, University of Bedfordshire, Milton Keynes, United Kingdom \\ *Corresponding author: Vseteckova J, Wellbeing and Social Care, Faculty of Wellbeing, Education and Language Studies, \\ University of Bedfordshire, Milton Keynes, United Kingdom
}

\section{ARTICLE INFO}

Received: 蔧 September 13, 2019

Published: 慧 September 25, 2019

\section{ABSTRACT}

Citation: Mahon A, Vseteckova J, Tilley E, Pappas Y and Randhawa G. A Systematic Scoping Review of the Impact of Mutual Care on Individuals with Learning Disabilities and their Ageing Carers in the United Kingdom. Biomed J Sci \& Tech Res 21(4)-2019. BJSTR. MS.ID.003621.

\section{Introduction}

The majority of individuals with Learning Disabilities (LD) within the United Kingdom (UK) are cared for by family members, usually a parent. As people with LD are now living longer than ever before this has resulted in the average age of carers for adults with LD also increasing, currently averageing 70 years of age or older [1]. Crucially, ageing carers present with care and support needs of their own. This has resulted in an increase of mutual care relationships. Mutual care relationships occur when an individual being cared for i.e. an individual with LD, takes on some level of care for their main carer. Mutual care relationships can become even more complex within these populations due to the co-morbidities associated with learning disabilities and ageing [1]. For example, individuals with LD are more likely to develop conditions related to older age - such as osteoporosis and diabetes - at a younger age compared to the general population [1] and are at a "greater risk of developing dementia than the general population $(22 \%$ vs $6 \%$ aged 65 and above) [2]. While the number mutual care relationships within the UK is predicted to rise, the impact of mutual care on the physical and mental wellbeing of both ageing carers and individuals with LD who assume a caring role remains unclear. A preliminary search for existing scoping reviews and/or systematic reviews on the topic was conducted. Within the UK there are limited primary research papers and research syntheses available on this topic. To the authors' knowledge no existing scoping reviews or systematic reviews on this topic currently exist.
The aim of the current scoping review is to provide a greater understanding of the impact mutual caring may have on the physical and mental wellbeing of both ageing carers and individuals with LD who assume caring roles. This will include identifying existing interventions that support mutual caring within the United Kingdom as well as the barriers and facilitators that can influence access to interventions and the effectiveness of identified interventions. Following the PICO framework [3], this scoping review aims to synthetize existing knowledge, identify gaps in the literature and provide recommendations for future research, which may lead to improved support for mutual caring relationships and the potential to improve the physical and mental wellbeing of both ageing carers and individuals with LD who assume caring roles.

\section{Methods}

A systematic scoping review was deemed the most suitable method due to the research questions proposed by the current review and the literature it intended to include. The current review aimed to provide a greater understanding of the impact of mutual caring on the physical and mental wellbeing of both ageing carers and individuals with LD who assume caring roles, as well as identifying any forms of intervention available to support mutual caring. While systematic reviews answer questions relating to specific interventions, the strict parameters required by this methodology deem it inappropriate for the purpose of the 
current review. Crucially, systematic scoping reviews are designed to synthesize knowledge according to an exploratory research question [4] and may include a wide variety of different research methodologies in the literature it includes, allowing it to provide an overview of broad research fields such as that included in the current review [5]. Furthermore, in line with the aims of the current review, scoping reviews may include any gaps in current research, make recommendations for future research as well as mapping key concepts and bodies of literature [4]. It is important to note that while scoping reviews generally do not require quality assessments of literature included, they do share a similar methodology to systematic reviews and as such they are rigorous, transparent and methodical in their approach [6-8].

As an extension of the Preferred Reporting Items for Systematic Reviews and Meta-analysis (PRISMA) statement called the Preferred Reporting Items for Systematic Reviews and MetaAnalysis: extension for Scoping Reviews (PRISMA-ScR) is currently under development [10], the current scoping review followed the methodological framework proposed by Arksey and 0'Malley [6] as well as the amendments to this framework proposed by Levac, Colquhoun and O'Brien [10], the Joanna Briggs Institute [11] and the guidelines for best practices provided by Colquhoun et el [12]. The Joanna Briggs Institute (JBI) methodology for Scoping Reviews, will be "congruent with the PRISMA-ScR checklist" and "will assist in standardizing future scoping reviews". It has been recommended that scoping review protocols follow the relevant aspects of the Preferred Reporting Items for Systematic Reviews and Meta-analysis for Protocols (PRISMA-P) guidelines [13] thus, the PRISMA-P was used to draft this current protocol [12].

The Arksey and O'Malley's scoping review framework consists of five consecutive stages:

1. identifying the research question,

2. identifying relevant studies,

3. study selection,

4. charting the data,

5. collating, summarising and reporting results. Each stage is discussed in further detail below. The last optional stage, consultation, was not included in the current scoping review.

\section{Stage 1}

\section{Identifying the Research Question}

The aim of the current scoping review was to provide greater understanding of the impact of mutual caring on the physical and mental wellbeing of both ageing carers and individuals with LD who assuming caring roles, as well as identifying any forms of intervention available to support mutual caring. This included identifying the barriers and facilitators that can influence access to interventions and collecting knowledge about the reported outcomes of these interventions. Following the PICO framework, this scoping review aimed to synthetize existing knowledge, identify gaps in the literature and provide recommendations for future research, which may lead to improved support for mutual care relationships with the potential to improve the physical and mental wellbeing of ageing carers and individuals with LD who assume caring roles.

To meet these objectives, this review asked the following questions:

1. What impact does a mutual care relationship between an (unpaid) ageing carer and an individual with LD, have on the physical and mental wellbeing needs of the (unpaid) ageing carer?

2. What impact does a mutual caring relationship between an (unpaid) ageing carer and an individual with LD, have on the physical and mental wellbeing needs of the individual with LD who has assumed a caring role?

3. What interventions are available within the UK to support mutual care relationships between an ageing carer and an individual with LD who has assumed a caring role?

4. What are the barriers and facilitators affecting access to interventions that support mutual care relationships within the UK?

5. What outcomes are reported by interventions that support mutual care relationships within the UK?

\section{Stage 2}

\section{Identifying Relevant Studies}

As scoping reviews provide an overview of broad research fields [5], the current review used a wide and diverse search to source all relevant studies. This included a search of electronic databases, reference lists (ancestor searching), website organisations and conference proceedings. Articles and evaluation reports related to the topic of ageing carers of individuals with learning disabilities were identified through an initial exploratory online search using the electronic databases MEDLINE (PubMed) and CINAHL. The text words in the title and abstract of relevant retrieved papers were then analysed as well as the index terms used to describe the articles. All identified keywords and index terms were used to develop a rigorous search strategy that was undertaken across all included databases. The reference list of identified reports and articles was also searched for additional studies. The reviewers contacted authors of primary studies or reviews for further information, where relevant. The search was limited to literature written in English. No date restrictions were applied. The search strategy and details of the search dates can be found in Appendix 1.

\section{Inclusion Criteria}

\section{Types of Participants}

Eligible participants included:

a) Carers aged 65 and over for individuals (adults; aged $40+$ ) with learning disabilities (LD) who are in a mutual care 
relationship i.e., the individual with LD is also assuming a caring role for the ageing carer.

b) Carers must not be caring for the individual with learning disabilities as part of their professional vocation i.e. they will be unpaid parents, family members or friends.

c) Providers and/or stakeholders involved in the provision of services specifically related to supporting mutual care relationships.

\section{Concept}

a) Types of Outcome Measures: to be included in the review, records had to report at least one of the following outcome measures:

A. Primary Outcomes: The primary outcome of interest is the impact of mutual caring on the physical and mental wellbeing of both ageing carers for individuals with learning disabilities as well as individuals with LD who have assumed a caring role, within the United Kingdom. It was expected that outcomes are diverse and context-specific, therefore, it was not possible to produce an exhaustive list at the outset. However, based on the review of the relevant literature, examples of primary outcomes regarding the impact of mutual caring on physical and mental wellbeing include:

i. Physiological impact (e.g. rate of co-morbid disease onsets, rate and/or progression of age or LD related physiological issues such as arthritis or joint replacements, changes in blood pressure and/or blood glucose level)

ii. Psychological impact (e.g. mental wellbeing including stress, depression, financial worries, fear of the future and anxiety, rate and/or progression of age or LD related issues such as dementia. These can be self-reported or measured using psychological assessment tools such as the Warwick-Edinburgh mental wellbeing scale (WEMWBS) and the Hospital Anxiety \& Depression scale (HAD))

iii. Social impact (e.g. social isolation, lack of new friendships and/or sense of belonging and/or independence)

B. Secondary Outcomes: The secondary outcomes of interest are the availability of interventions that support mutual caring, including identifying the facilitators and barriers affecting access, and the outcomes for service users, including personal, social, wellbeing, and health-related outcomes. Any interventions or any form of support available will be plotted in accordance with the support they provide (i.e. physiological, psychological or social). Facilitators and barriers to accessing interventions may include: availability of interventions, awareness of available resources, how services and service providers are perceived, trust of the service user in the service provider, concerns regarding the care needs being met, concerns that care will be transferred outside of the home, issues with finance or transport. The effectiveness of interventions in supporting mutual care relationships may be assessed according to:

i. Physiological support (e.g. any reported changes in agerelated disease or physiological issues, blood pressure and/or blood glucose level, provision of external support e.g., walking aids, support workers, physical rehabilitation.)

ii. Psychological support (e.g. any reported changes in mental wellbeing including stress, depression, financial worries, fear of the future, anxiety and perception of care providers. These can be self-reported or measured using psychological assessment tools)

iii. Social support (e.g. any reported changes in social isolation, friendships and/or sense of belonging and/or independence).

Types of interventions: The aim of this systematic scoping review is to synthesize knowledge regarding all available information related to the exploratory research questions, as such all interventions will be included.

Context/Setting: All participants must live within the United Kingdom. If the participants did not live in the United Kingdom they were excluded. Interventions that were implemented in the UK were included in the review. Those interventions that were implemented outside of the UK were excluded.

\section{Types of Studies}

The purpose of the current scoping review was to synthesize all relevant available knowledge. To provide a comprehensive overview of this research topic all existing literature was included, e.g. primary research studies, systematic reviews, meta-analyses, letters, guidelines, websites etc.

\section{Electronic Searches}

The following electronic databases were searched:

a) CINAHL

b) British Nursing Index

c) Web of Science

d) Cochrane library

e) Medline

f) Psych Info

g) SocIndex

h) University of York Centre for Reviews and Dissemination (DARE, NHS EED, HTA)

i) JBI Database of Systematic Reviews and Implementation Reports,

j) PubMed 

k) EPPI
l) Epistemonikos,

To identify relevant evaluations in UK settings, the websites of the following organisations were searched:
i. The Kings Fund
ii. The Health foundation
iii. NESTA
iv. NICE
v. Nuffield Trust
vi. Department of Health

Additionally, grey literature was searched in Open Grey, Google, and Google Scholar. The search terms "mutual care ageing carers of learning disabilities" and "co-caring older carers of learning disability" were used to identify grey literature because they were identified as the most relevant terms in the exploratory and database searches.

Searching other Resources: In addition, reference lists of all relevant studies, reviews, and reports were searched. Via contacting the British Library and other University libraries, the researcher obtained a full text PDF or an abstract containing sufficient details to determine eligibility of all potentially relevant studies.

\section{Stage 3: Study Selection}

\section{Study Screening and Selection}

Study selection (both at title/abstract screening and full text screening) was performed by three reviewers, independently. Any disagreements were solved by consensus or by the decision of a fourth reviewer were necessary. After eliminating the duplicates (studies that were identified more than once by the search engines), an initial screening of titles, abstracts, and summaries (if applicable) was undertaken to exclude records that clearly did not meet the inclusion criteria. Each record was classified as 'include' or 'exclude' to identify relevant and exclude irrelevant literature. The researchers were inclusive at this stage and, if uncertain about the relevance of a publication or report, it was left in. The full text was obtained for all the records that potentially met the inclusion criteria (based on the title and abstract/summary only). In a second step, all the full text papers were screened against the inclusion criteria, using a standardised tool. Studies that did not meet the inclusion criteria were listed with the reasons for exclusion. Multiple publications and reports on the same interventions were linked together and compared for completeness. The record containing the most complete data on any single intervention was identified as the primary article in the review, which was usually the original study or most recent evaluation report. An adapted PRISMA (Preferred Reporting Items for Systematic Reviews and Meta-analyses) flow-chart of study selection was included in the review [14].

\section{Stage 4: Charting the Data \\ Data Extraction and Management}

Data for analysis were extracted from the included studies and managed in an Excel spread sheet. A data extraction sheet was developed which was tailored to the requirements of the review. The data extraction sheet was tested on three included papers and, where necessary, it was revised to ensure it can be reliably interpreted and could capture all relevant data from different study designs. Extracted data included authors, year of study/ report, aim/purpose, type of paper (e.g. journal article, annual evaluation report, etc), geographical area, study population (e.g., age of participants, learning disability diagnosed, any additional co-morbid diagnosis), sample size, study design, identified mental wellbeing impacted needs (psychological and social), identified physical wellbeing impacted needs, description of the interventions/services/support for mental wellbeing needs, description of the interventions/services/support for physical wellbeing needs, duration of interventions, factors that facilitate and/or hinder access to interventions/services/support, outcomes reported by service users accessing/not accessing interventions (and how measured), key findings that relate to the scoping review questions.

\section{Stage 5: Collating, Summarising and Reporting the} Results

\section{Presentation of the Results (Data synthesis)}

Findings from included studies were synthesised narratively. The 'Guidance on the Conduct of Narrative Synthesis in Systematic Reviews' was used to advise the narrative synthesis [15]. First, a preliminary synthesis was conducted to develop an initial description of the findings of included records and to organise them so that patterns across records could be identified. In a second step, thematic analysis was used to analyse the findings. The following five steps of thematic analysis were followed adopting a recursive process [16]:

1. Familiarisation with the extracted data

2. Generation of initial codes

3. Searching for themes

4. Reviewing themes

5. Defining and naming themes

Depending on the findings available the reviewers will aim to provide a flow chart mapping the interventions available for ageing carers of individuals with learning disabilities, for the needs that were identified.

\section{References}

1. (2015) National Institute for Health and Care Excellence. Equality impact assessment: NICE Guidelines on the Care and Support of Older People with Learning Disabilities (LD). 
2. Bennett L (1998) British Institute of Learning Disabilities (LD). Learning Disability Practice1(2): 4-5.

3. Richardson WS, Wilson MC, Nishikawa J, Hayward RS (1995) The wellbuilt clinical question: a key to evidence-based decisions. ACP journal club 123(3): A12.

4. Colquhoun HL, Levac D, O’Brien KK, Straus S, Tricco AC, et al. (2014) Scoping reviews: time for clarity in definition, methods, and reporting. J Clin Epidemiol 67(12):1291-1294.

5. Moher D, Shamseer L, Clarke M, Ghersi D, Liberati A, et al. (2015) Preferred reporting items for systematic review and meta-analysis protocols (PRISMA-P) 2015 statement. Systematic reviews 4(1): 1.

6. Arksey H, O'Malley L Scoping (2005) studies towards a methodological framework. Int J Soc Res Methodol 8(1):19-32.

7. Halas G, Schultz AS, Rothney J, Goertzen L, Wener P, et al. (2015) A scoping review protocol to map the research foci trends in tobacco control over the last decade. BMJ open 5(1): e006643.

8. Pham MT, Rajić A, Greig JD, Sargeant JM, Papadopoulos A, et al. (2014) A scoping review of scoping reviews: advancing the approach and enhancing the consistency. Research synthesis methods 5(4): 371-85.

9. Tricco A, Strauss S, Moher D (2015) Preferred Reporting Items for Systematic Reviews and Meta-Analysis: extension for Scoping Reviews (PRISMA-ScR). Equator Network.

\section{ISSN: 2574-1241}

DOI: 10.26717/BJSTR.2019.21.003621

Vseteckova J. Biomed J Sci \& Tech Res

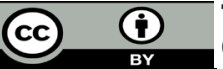

This work is licensed under Creative Commons Attribution 4.0 License

Submission Link: https://biomedres.us/submit-manuscript.php
10. Levac D, Colquhoun H, O Brien KK (2010) Scoping studies: advancing the methodology. Implement Sc 5: 69 .

11. (2015) Joanna Briggs Institute. The Joanna Briggs Institute reviewers' manual 2015: methodology for JBI scoping reviews. Adelaide, Australia: University of Adelaide.

12. Colquhoun H Current (2016) Best Practices for the Conduct of Scoping Reviews.

13. Shamseer L, Moher D, Clarke M, Ghersi D, Liberati A (2015) Preferred reporting items for systematic review and meta-analysis protocols (PRISMA-P) elaboration and explanation. Bmj 349: g7647.

14. Moher D, Liberati A, Tetzlaff], Altman DG (2009) Prisma Group. Preferred reporting items for systematic reviews and meta-analyses: the PRISMA statement. PLoS medicine6(7): e1000097.

15. Popay J, Roberts H, Sowden A, Petticrew M, Arai L, et al. (2006) Guidance on the conduct of narrative synthesis in systematic reviews. A product from the ESRC methods programme Version 1: b92.

16. Braun V, Clarke V (2006) Using thematic analysis in psychology. Qualitative research in psychology 3(2): 77-101.

$\begin{array}{ll}\text { BIOMEDICAL } & \text { Assets of Publishing with us } \\ \text { RESEARCHES } & \text { - Global archiving of articles } \\ & \text { - Immediate, unrestricted online access } \\ \end{array}$

